\title{
ADAM17 overexpression promotes angiogenesis by increasing blood vessel sprouting and pericyte number during brain microvessel development
}

\author{
JUNTANG LIN ${ }^{1}$, CORNELIUS LEMKE ${ }^{1}$, CHRISTOPH REDIES ${ }^{1}$, XINYAN², \\ EILHARD MIX ${ }^{2,3}$, ARNDT ROLFS ${ }^{2}$ and JIANKAI LUO*,1,2 \\ ${ }^{1}$ Institute of Anatomy I, School of Medicine University of Jena, Jena, ${ }^{2}$ Albrecht-Kossel-Institute for Neuroregeneration \\ and ${ }^{3}$ Department of Neurology, School of Medicine, University of Rostock, Germany.
}

\begin{abstract}
The angiogenic process is precisely regulated by different molecular mechanisms, with a balance between stimulatory and inhibitory factors in embryonic development. Transmembrane proteins of the ADAM (a disintegrin and metalloprotease) family play a critical role in embryogenesis and are involved in protein ectodomain shedding, as well as cell-cell and cell-matrix interactions. In the present study, we found that ADAM17 is expressed spatiotemporally in the tectal layers during chicken embryonic development. To investigate the effect of ADAM17 overexpression on angiogenesis, chicken ADAM17 plasmids were transfected into the developing tectum in vivo by electroporation. Results showed that overexpression of ADAM17 induces morphological changes of brain microvessels, such as an increase in diameter, of capillary sprouting from radial microvessels and an increase in the number of pericytes, but not of endothelial cells. Our data suggest that overexpression of ADAM17 in the developing tectum promotes angiogenesis by increasing the number of pericytes and capillary sprouting in the radial vessels.
\end{abstract}

KEY WORDS: ADAM17, angiogenesis, brain development, microvessel, pericyte

\section{Introduction}

During brain development, the microvessels inside the brain are derived from the leptomeningeal vessels by a sprouting angiogenic process (Risau, 1997). The sprouting capillaries penetrate into the brain, where the extracellular matrix is degraded. The wall of a typical brain microvessel is principally composed of two distinct cell types: endothelial cells and pericytes. The endothelial cells form the inner side of the vascular tube and the pericytes form an outer sheath surrounding the endothelium (Nehls et al., 1992; Risau, 1997). The endothelial cells of brain microvessels connect to each other by tight junctions and interact directly with numerous pericytes in order to form the highly impermeable blood-brain barrier, which protects brain cells from potential toxic substances residing in the systemic blood circulation (Lai and Kuo, 2005). Pericytes are essential for the formation, maturation and maintenance of the normal microvascular structure and function (Bergers and Song, 2005). Angiogenesis is a complex process that includes the generation of space for cell migration in tissue by removal of obstructing matrix proteins, the degradation of endothelial basement membranes, and the modification of growth factors, cytokines and receptors
(Patan, 2000; van Hinsbergh and Koolwijk, 2008).

ADAM proteins are involved in cell-cell and/or cell-matrix interactions and mediate potentially protease activities, cell adhesion and cell signal transduction (Blobel, 2005; Edwards et al., 2009). For example, ADAM10 and ADAM17 have been identified as major sheddases for the ligands of the epidermal growth factor receptor (EGFR) and Notch. The activation of EGFR and Notch signaling pathways contribute to physiological and pathological events, e.g., to angiogenesis in developing embryos and tumor growth (Sahin and Blobel, 2007; Edwards et al., 2009).

Interestingly, ADAM17 is expressed by endothelial cells and astrocytes in the adult human brain (Goddard et al., 2001). Deletion of ADAM17 in cultured endothelial cells inhibits cell proliferation

\footnotetext{
Abbreviations used in this paper: ADAM, a disintegrin and metalloprotease; EGFR, epidermal growth factor receptor; GFP, green fluorescence protein; PGNA, proliferating cell nuclear antigen; SAC, stratum album centrale; SFP, stratum fibrosum periventriculare; SGC, stratum griseum centrale; SGFS, stratum griseum et fibrosum superficiale; SGP, stratum griseum periventriculare; SMA, alpha smooth muscle actin. SO, stratum opticum; TNF-alpha, tumor necrosis factor type alpha; VEGF, vascular endothelial growth factor.
}

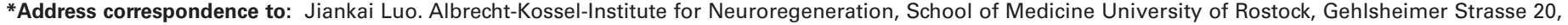
D-18147 Rostock, Germany. Tel: +49-381-4949-629. Fax: +49-381-4944-899. e-mail: jiankai.luo@uni-rostock.de
} 
and capillary-like structure formation (Göoz et al., 2009). In human primary colon carcinoma, ADAM17 is over- and co-expressed with the EGFR in both neoplastic and endothelial cells, suggesting that ADAM17 may induce tumor growth by promoting angiogenesis in tumor tissues (Edwards et al., 2009).

In the present study, we used the chicken embryo as a model to investigate the effect of ADAM17 on angiogenesis of brain microvessels. By ex ovo electroporation, plasmids encoding ADAM17 were transfected into the developing tectum at embryonic incubation day (E) 6 and their overexpression and angiogenic effect were investigated from E10 to E14. Our results show that the overexpression of ADAM17 has a strong effect on the morphology and cellular composition of brain microvessels in the developing brain.

\section{Results}

\section{Expression of ADAM17 mRNA during chicken tectal develop- ment}

To monitor the transcription of ADAM17 mRNA during chicken embryonic development, we performed a semi-quantitative RT-PCR analysis using embryos from E1.5 to E20 (Fig. 1 A,B). The results showed that ADAM17 is transcribed from E1.5 to E4 in whole embryos. In the head, the expression of ADAM17 is kept at a high level from E5 to E8. In the brain, ADAM17 expression is strong from E10 to $\mathrm{E} 14$ and becomes slightly weaker from E16 onward. There is no band found in the negative control (Fig. 1A). These results suggest that the expression of ADAM17 mRNA is maintained during the entire development of the chicken embryo.

During development of the chicken tectum, cells in the subventricular zone migrate after mitosis radially into the mantle layer, where they differentiate into neurons and form a laminated structure (Domesick and Morest, 1977). The mature chicken tectum can be divided into six major layers (from pia to ventricle): the stratum op- ticum (SO), the stratum griseum et fibrosum superficiale (SGFS), the stratum griseum centrale (SGC), the stratum album centrale (SAC), the stratum griseum periventriculare (SGP), and the stratum fibrosum periventriculare (SFP) (LaVail and Cowan, 1971). The mature tectal structure can be recognized at about E14 by thionine staining (Fig. 1J).

Next, we investigated the expression of ADAM17 mRNA in frontal sections of the chicken tectum from E6 to E20 by in situ hybridization (Fig. $1 \mathrm{C}-\mathrm{H}$ ). Sense ADAM17 probe was used as a negative control (Fig. 1I). At E6, the neuroepithelial cells within the ventricular layer express ADAM17 mRNA abundantly (Fig. 1C). At E8, the cells within SGFC start to transcribe ADAM17 (Fig. 1D). At E10, the cells within SGC gradually become ADAM17 positive (Fig. $1 \mathrm{E})$. Gradually from E14 to E20, ADAM17 expression in the cells of the inner layers of SFP, SGP and SGC becomes stronger while expression in the cells of the outer layer of SGFC becomes weaker (Fig. $1 \mathrm{~F}-\mathrm{H}$ ). Therefore, the temporal and spatial transcription of ADAM17 in the different tectal layers suggests a role of ADAM17 during tectal development. It should be noted that we have not found any endogenous transcription of ADAM17 in the radial microvessels of the tectum (Fig. $1 \mathrm{C}-\mathrm{H}$ ).

\section{Overexpression of ADAM17 enlarges radial microvessels and promotes capillary sprouting from radial microvessels}

During chicken tectal development, microvessels derived from the leptomeningeal vessels start to penetrate optic tectal tissue at about E5 (Roncali et al., 1986; Risau, 1986). To study blood vessel formation, we used the following markers: fibronectin (Fn), a specific marker for vascular extracellular matrix during chicken embryonic development (Fig. 2B) (Risau and Lemmon, 1988); alpha smooth muscle actin (SMA), a marker for pericytes and smooth muscle cell, which has been used as a specific pericyte marker during chicken embryonic brain development (Fig. 2C) (Skalli et al., 1986; Gerhardt
A
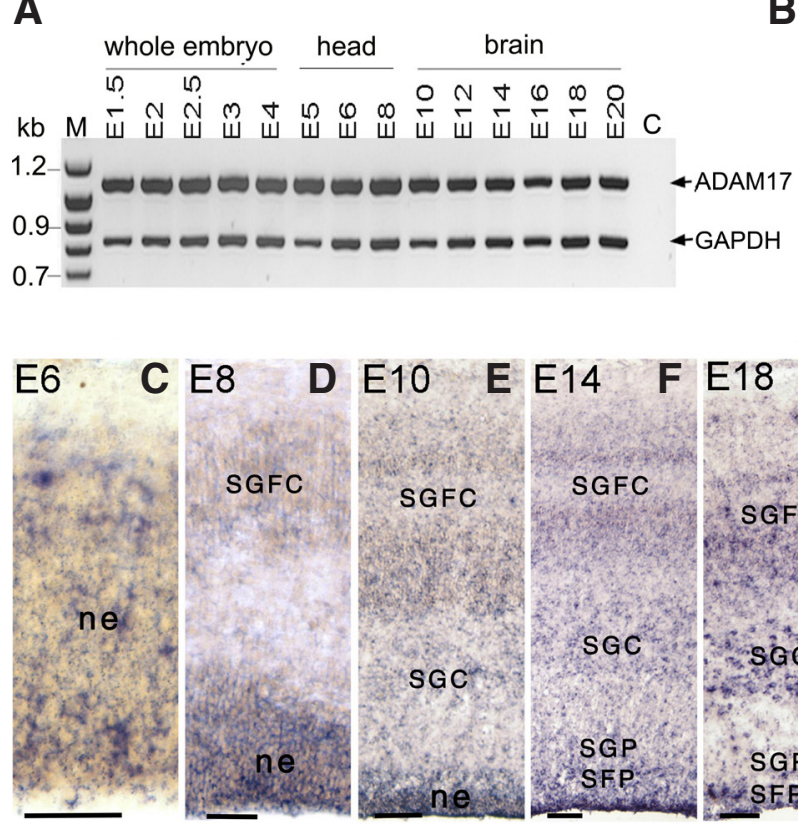
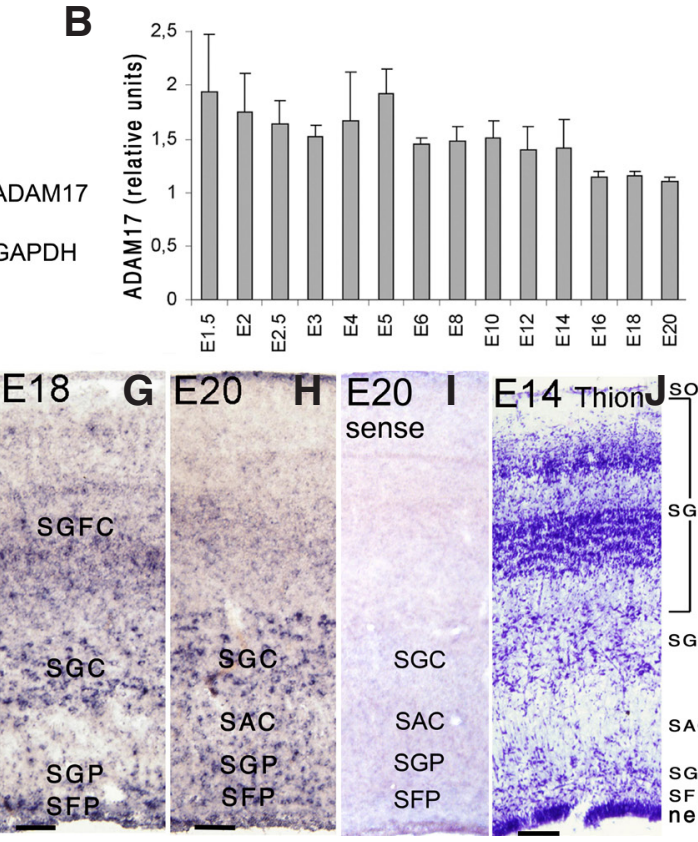

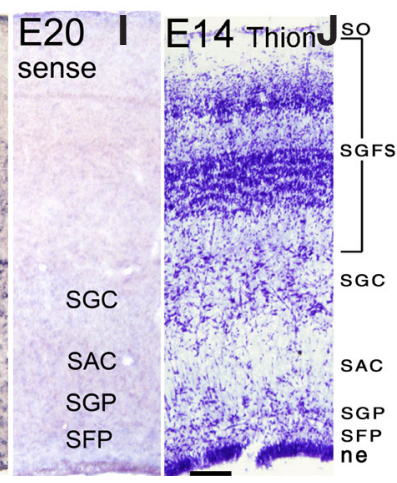

Fig. 1. Analysis of ADAM17 transcription in the developing chicken embryo by semiquantitative RT-PCR and in situ hybridization. (A,B) Semiquantitative RT-PCR analysis of ADAM17 at different days of incubation (E) (E1.5-E4, whole embryos; E5-E8, whole head; E10-E20, brain). Glyceraldehyde3-phosphate dehydrogenase (GAPDH) is used as an internal control to monitor the amount of RNA. Water or reverse transcriptase free reaction was used as a negative control. Values are presentedas means $\pm S D$. (C-J) Expression of ADAM17 mRNA in frontal sections of tectum at different days of incubation. Sense ADAM17 probe was used as a negative control (I)

Tectal layers can be identified in thionine stains ( $\mathbf{J})$ and are marked in (C-J). The pial surface is on the top and the ventricular layer is on the bottom. ne: neuroepithelium; SAC: stratum album centrale; SFP: stratum fibrosum periventriculare; SGC: stratum griseum centrale; SGFC: stratum griseum et fibrosum superficiale; SGP: stratum griseum periventriculare; SO: stratum opticum. Scale bars: $50 \mu \mathrm{m}$ in (C-E), $100 \mu \mathrm{m}$ in (F, G, J) in (H) for (H and I). 
etal., 2000); HT7, a specific marker forendothelial cells in blood-brain barrier (Fig. 2D) (Bertossi et al., 2002); and laminin (3H11 and 31), a major basement membrane component of blood vessels (Kukita et al., 1998). We confirmed that the radial microvessels are found in the tectum at E12 (arrows in Fig. 2 B-D). During the elongation of the radial microvessels to the inner part near the ventricular layer, a few blood vessels sprout out from the radial microvessels and form the inner vascular plexus inside the tectum (data not shown).

To investigate the effect of ADAM17 overexpression on the development of brain microvessels, ADAM17 and/or GFP plasmids were
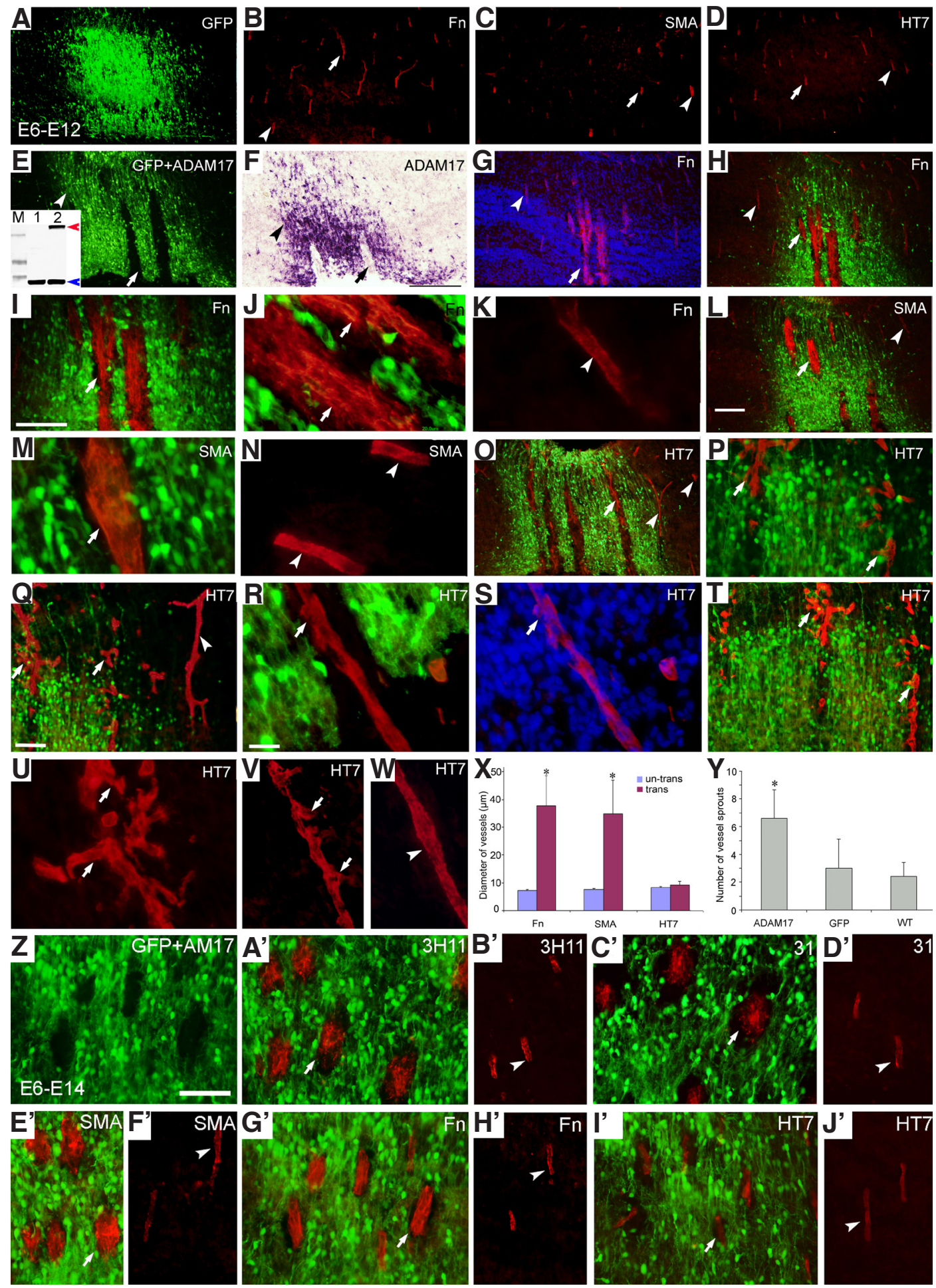

Fig. 2. Overexpression of ADAM17 in developing tectum promotes angiogenesis. (A-D) Sections from embryos transfected with GFP alone at E6 were investigated at E12 by immunostaining against fibronectin (red in B), SMA (red in C), and $H T 7$ (red in D. The transfected region is indicated by GFP-positive cells (green in A). The radial microvessels inside the transfected region are indicated by arrows and outside by arrowheads. (E-W) Sections from embryos transfected with ADAM17 at E6 were investigated at E12 by in situ hybridization (purple in F) or immunostaining for $F n$ (red in G-K), SMA (red in L-N), and HT7 (red in O-W). The transfected regions are indicated by GFP-positive cells (green, E-T). The insert in $E$ shows ADAM17 expression (red arrowhead) in the lysates of human HEK cell transfected with chicken pCAGGS-ADAM17 (lane 2), but not with pCAGGS-GFP (lane 1) by Western blotting. GAPDH is used as a loading control (blue arrowhead). The arrows (E-J, L, M, $\mathbf{O}-\mathbf{V})$ indicate the radial microvessels inside the transfected regions, while, the arrowheads (E-Q) indicate the radial microvessels outside the transfected regions. Cell nuclei are stained with Hoechst 33258 or DAPI (G,S). Scale bars: 50 um in (I) for (I-K, M,N), in $(Q)$ for $(P)$, in (R) for $(R-W) ; 100$ $\mu \mathrm{m}$ in (L) for (A-H, O). (X) The size of the radial microvessels revealed by immunohistochemistry against fibronectin (Fn), alpha-smooth muscle actin (SMA) and HT7 antibodies are quantitatively analyzed in the inside (trans) and the outside (un-trans) of the transfected regions at E12 after electroporation at E6. * $\mathrm{P}<0.05$. (Y) Quantitative analysis of the numbers of vessels sprouting from the radial microvessels at E12 in the groups of transfection with ADAM17 or GFP at E6 or wild type (WT) without electroporation. Values are presented as means \pm $S D,{ }^{*} \mathrm{P}<0.05$. (Z-J') Sections from embryos transfected with ADAM17 at E6 were investigated at E14 by immunostaining for $3 H 11$ (red in $\left.A^{\prime}, B^{\prime}\right), 31$ (red in $C^{\prime}$ and $D^{\prime}$ ), SMA (red in $\left.E^{\prime}-F^{\prime}\right)$, Fn (red in $\left.G^{\prime}-H^{\prime}\right)$, and HT7 (red in $\left.I^{\prime}-J^{\prime}\right)$. The transfected regions are indicated by GFP-positive cells (green, $Z$, $A^{\prime}$, $\left.C^{\prime}, E^{\prime}, G^{\prime}, I^{\prime}\right)$. Scale bars: $50 \mu \mathrm{m}$ in $Z$ for Z-J'. 
transfected into the tectal neuroepithelial cells by ex ovo electroporation at E6. Co-electroporation of two plasmids into chicken embryo by ex ovo electroporation results in a high level of coexpression of the two proteins encoded by the transfected plasmids (Luo et al., 2004, 2006; Luo and Redies, 2005). Therefore, in this study, GFP plasmid was cotransfected and used as a marker to trace the cells expressing ADAM17 ectopically. To test whether ADAM17 construct can express ADAM17 protein, pCAGGS-ADAM17 plasmid was transfected into cultured human HEK cells in vitro. Result showed that ADAM17 protein was found in the cell lysate by Weretern blotting (red arrowhead, lane 2 in the insert of Fig. 2E), suggesting that PCAGGS-ADAM17 can be used for ADAM17 overexpression in vivo by electroporation.

When pCAGGS-GFP plasmid was transfected alone into the tectum at $E 6$ (control electroporation), the radial microvessels inside (arrows in Fig. 2 B-D) and outside (arrowheads in Fig. 2 B-D) the transfected regions (green in Fig. 2A) are not different at E12 (Fig. $2 A-D$ ) and E14 (data not shown) by immunostaining with fibronectin (Fig. 2B), SMA (Fig. 2C) and HT7 antibodies (Fig. 2D).

When pCAGGS-ADAM17 was cotransfected together with pCAGGS-GFP plasmid, at E12 some large GFP-negative columns (arrow in Fig. 2E) were found inside the transfected regions (green, arrowhead in Fig. 2E), where the radial microvessels penetrate into the optic tectal tissue (arrow in Fig. 2G). ADAM17 mRNA is transcribed strongly in the transfected region of the adjacent sections (arrowhead in Fig. 2F), but not by the penetrating radial microvessels, as visualized by in situ hybridization (arrow in Fig. 2F) in the transfected regions of adjacent sections. GFP expression is absent from blood vessels, suggesting that blood vessels are not electroporated at this stage (Fig. 2E). The same results were also obtained at E14 (Fig. 2Z).
At E12, by immunohistochemistry against fibronectin (Fig. 2 $\mathrm{H}-\mathrm{K}$ ) and SMA (Fig. $2 \mathrm{~L}-\mathrm{N}$ ), we found that the radial microvessels (arrows in Fig. $2 \mathrm{H}-\mathrm{J}, \mathrm{L}, \mathrm{M}$ ) inside the ADAM17-transfected regions (green in Fig. $2 \mathrm{H}-\mathrm{J}, \mathrm{L}, \mathrm{M}$ ) are much larger than those outside the transfected regions (arrowheads in Fig. $2 \mathrm{H}, \mathrm{K}, \mathrm{L}, \mathrm{N}$ ). To estimate the morphological change of the radial microvessels in transfected regions, we counted the size of the radial vessels in the images stained with fibronectin and SMA by Keyence Analyser System (BZ-8000; Keyence Deutschland GmbH, Neu-Isenburg, Germany). Results showed that the size of the radial microvessels inside the transfected regions is about three times larger than those outside the transfected regions and the difference between them is significant (Fig. 2X). However, immunostaining results for HT7 (Fig. 2 O-W) demonstrated that the diameter of the endothelial tube of the radial microvessels is similar inside (arrows in Fig. $2 \mathrm{O}-\mathrm{T}$ ) and outside (arrowhead in Fig. $2 \mathrm{O}-\mathrm{Q}, \mathrm{W}$ ) of the transfected regions (Fig. 2X).

At E14, when the blood-brain barrier is full developed in the chickenoptic tectum (Roncali etal., 1986), the immunohistochemistry results of the radial microvessels were found to be similar to those at E12 using the antibodies against fibronectin, SMA, laminin, and HT7 when ADAM17 was overexpressed in the developing tectum. The microvessels inside the ADAM17-transfected regions stained with antibodies against laminin (3H11 and 31; Fig. 2 A', C'), SMA (Fig. 2E') and fibronectin (Fig. 2G') are much larger than those in the un-transfected regions (Fig. 2 B', D',F',H'); But the microvessels stained with HT7 at E14 inside the transfected regions (Fig. 2l') are similar to that outside the transfected regions (Fig. 2J'). Take together, our data suggest the possibility that the enlargement of the radial microvessels in the electroporated area is due to an increase in the amount of extracellular matrix deposition in the microvessels and/ or a larger number of the pericytes.
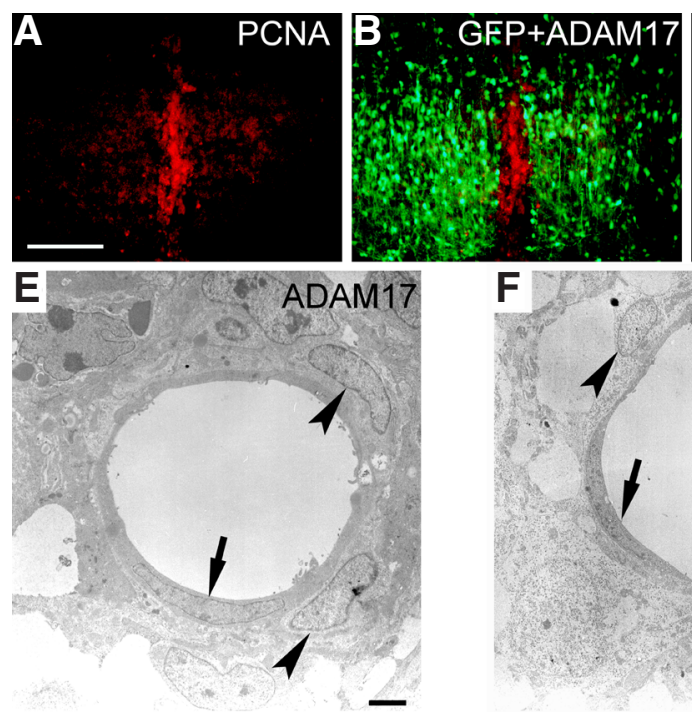

H

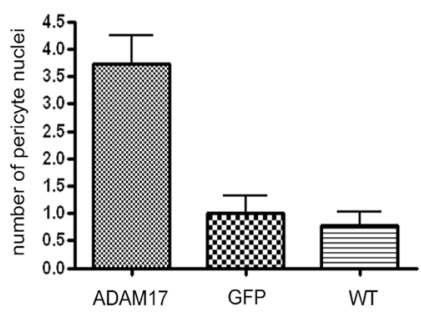

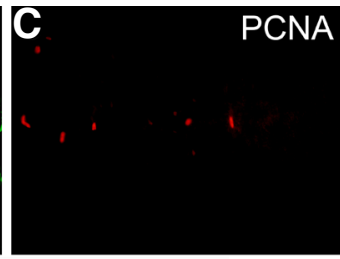
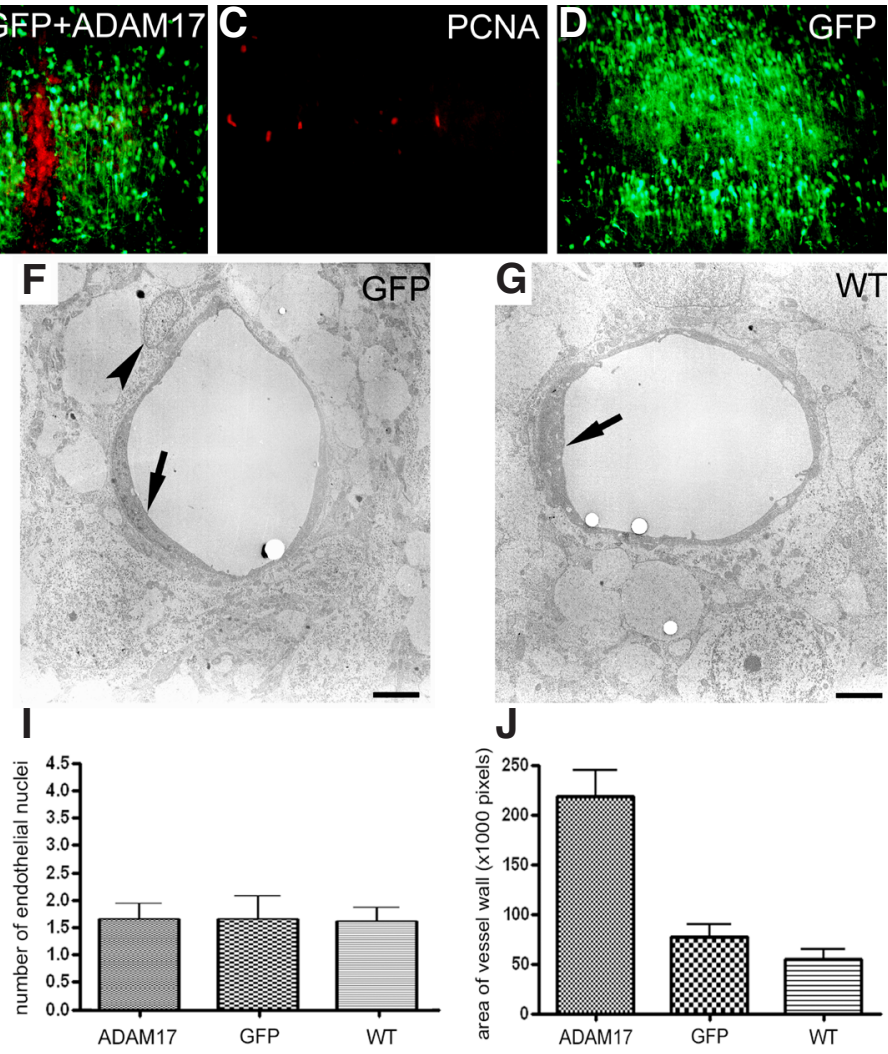

G

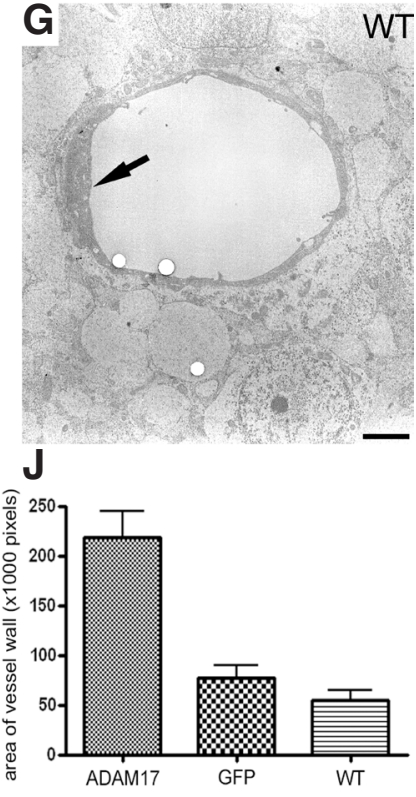

Fig. 3. Cell proliferation assay, digital images and quantitative analysis of radial microvessels by transmission electron microscopy. (A-D) Sections of tectum from embryos transfected with ADAM17 $(A, B)$ or GFP alone $(C, D)$ at E6 were investigated at $E 12$ by immunostaining with PCNA red color in A and C). The pial surface is on the bottom and the ventricular zone is on the top. Scale bar: $100 \mu \mathrm{m}$ in A for A-D. (E-G) Digital images of radial microvessels in frontal section of tectum at E12 after transfection with ADAM17 (E) or GFP (F) at E6 or without electroporation in wild type tectum (WT; G). The arrows indicate the endothelial cells and the arrowheads indicate the pericytes. Scale bars: $2 \mu \mathrm{m}$ in D; $2.5 \mu \mathrm{m}$ in $E ; 3$ um in F. (H-J) Quantitative analysis of the nucleinumbers of the pericytes $(H)$ and the endothelial cells (I) around the radial microvessels and of the area occupied by the radial microvesse/ walls (J) at E12 in the groups of transfection with ADAM17 or GFP at E6 or wild type (WT) without electroporation. Values are presented as means \pm SEM, ${ }^{*} \mathrm{P}<0.05$. 
Furthermore, by immunostaining with HT7 antibody (Fig. 2 Q-W), we found that the radial microvessels inside the ADAM17transfected regions are characterized by a large number of capillary sprouts (arrows in Fig. $2 \mathrm{Q}-\mathrm{V}$ ), if compared to the vessels outside the transfected region (arrowheads in Fig. 2 Q,W). We therefore also counted the number of capillary sprouts from the radial microvessels in the experimental and control groups (ADAM17 electroporation, GFP electroporation alone, and no electroporation, respectively). The results showed that the number of the capillary sprouts from radial microvessels in the ADAM17-transfected group is significantly increased (Fig. 2Y), suggesting that ADAM17 overexpression promotes capillary sprouting during tectal development.

Next, we asked whether the size enlargement of radial microvessels and the increase of the capillary sprouts under ADAM17 overexpression may be due to apoptosis of neuronal precursors induced by the electroporation procedure. Results from the TUNEL assay revealed that neuronal apoptosis is not significantly different between the three distinct groups (data not shown).

\section{ADAM17 overexpression promotes pericyte proliferation}

To investigate the mechanism of the enlargement of radial microvessels induced by ADAM17 overexpression, we evaluated cell proliferation in the sections of the GFP- and ADAM17-transfected embryos using an antibody raised against proliferating cell nuclear antigen (PCNA). The PCNA-positive cells in radial microvessels were found mainly inside the ADAM17-transfected regions (Fig. 3 A,B), but few inside the GFP-transfected regions (Fig. 3 D,C), suggesting that the overexpression of ADAM17 promotes cell proliferation in the radial microvessels.

Furthermore, we analyzed the size and cellular components of the radial microvessels by electron microscopy. We chose the digital images of the radial microvessels in three different groups at random. The number of pericyte nuclei and endothelial cell nuclei were counted according to their location and morphological shape, and the area of the vessel walls, as indicated by number of pixels, was measured using the ImageJ program. Results demonstrate that the number of pericytes (arrowheads in Fig. $3 \mathrm{E}-\mathrm{G}$ ) around the endothelial cells (arrows in Fig. 3E-G) was significantly increased in the radial microvessels of the ADAM17-transfected group (Fig. $3 \mathrm{E}, \mathrm{H}$ ) compared to the controls (GFP transfection, Fig. $3 \mathrm{~F}, \mathrm{H}$; and untransfected wild type, Fig. $3 \mathrm{G}, \mathrm{H}$ ). In contrast, the number of the endothelial cells did not differ significantly between the three groups (Fig. 3 E-G,I). However, the area occupied by the radial microvessel wall was significantly larger in the ADAM17-transfected group than that in the two control groups (Fig. $3 \mathrm{E}-\mathrm{G}, \mathrm{J})$, but the inner diameters of the radial microvessels did not differ significantly between the three groups (Fig. 3 E-G). Therefore, the enlargement of the radial microvessels induced by ADAM17 overexpression results from an increased thickness of the vessel wall and is due to a higher number of pericytes.

In summary, our data suggest that ADAM17 overexpression promotes angiogenesis during tectal development by enhancing capillary sprouting from the radial microvessels and increasing pericyte number and vessel wall thickness.

\section{Discussion}

In the present study, we investigated the in vivo effect of ADAM17 overexpression on angiogenesis using the developing chicken em- bryo as a model. Although ADAM17 is not expressed endogenously by brain microvessels, the overexpression of ADAM17 in developing brain promotes angiogenesis by increasing capillary sprouting and pericyte proliferation (Figs. 2-3).

The angiogenic process is precisely regulated at distinct stages by different molecular mechanisms with a balance between stimulatory and inhibitory factors (Risau, 1997; Patan, 2000). Proteins of the ADAM family regulate the processes of angiogenesis by remodeling cell migration and invasion, and the liberation and modification of growth factors (van Hinsbergh and Koolwijk, 2008).

Interestingly, ADAM17 is important for the vascular endothelial growth factor (VEGF) receptor 2 stimulated processing of several receptors with known functions in endothelial cell biology (Weskamp et al., 2010). An ADAM17-dependent crosstalk between the VEGF receptor 2 and the extracellular signal-regulated kinase (ERK) signaling contributes angiogenesis (Swendeman et al., 2008). Loss of ADAM17 activity in the mutant-mouse impairs embryonic vessel formation and causes hemorrhage (Canault et al., 2010). Furthermore, tumor necrosis factor type alpha (TNF-alpha) - a target substrate of ADAM17, stimulates vascular sprouting of blood vessels in the rabbit and rat cornea as well as the developing chicken chorioallantoic membrane in vivo, resulting in an increase of angiogenesis (Leibovich et al., 1987). Moreover, ADAM17 can shed the ligands of the EGFR and Notch, activating their signaling pathways and contributing to angiogenesis (Edwards et al., 2009). Therefore, it is possible that overexpression of ADAM17 in developing brain may help to promote angiogenesis by activating signal pathways of related growth factors and cytokines via, e.g., Notch and TNF-alpha.

The interaction between pericytes and endothelial cells plays an essential role for vascular development, stabilization, maturation and remodeling (Choi and Kim, 2008). Pericytes have been proposed to be involved in 1) sensing the presence of stimuli to angiogenesis;2) controlling endothelial proliferation and differentiation; and 3) depositing or degrading extracellular matrix proteins (Dore-Duffy, 2008). Several studies have shown that pericytes regulate development of angiogenic sprouts during the early phases of neovascularization in both physiological and pathological conditions (Nehls et al., 1992; Ozerdem and Stallcup, 2003). During human telencephalic development, activated pericytes are present as early as mitotic endothelial cells and form the endothelium-free pericyte tubes at an early stage of vessel growth. The endothelial cells of the radial vessels are recruited and guided by pioneering pericytes, suggesting that pericytes stimulate and guide endothelial cells to form new vessels (Ozerdem and Stallcup, 2003). Furthermore, a previous study demonstrated that the somata and processes of pericytes engulf the leading tips of vascular sprouts in the developing chicken ganglionic eminence at E5 (Gerhardt et al., 2000). Therefore, in the present study, ADAM17 overexpression in the tectum promotes the proliferation of pericytes around the radial microvessels (Figs. 2-3). On one side, the increased pericytes may produce humoral factors including VEGF which stimulate endothelial outgrowth, resulting in an increase of the capillary sprouting of the microvessels (Reinmuth et al., 2001). On the other side, the pericytes may inhibit the endothelial proliferation and control the capillary differentiation by cell-cell contacts (Nehls et al., 1992), avoiding the over-proliferation of the endothelia cells.

However, how ADAM17 overexpression in developing brain promotes the proliferation of pericytes in radial microvessels is 
unknown. Risau reported already in 1986 that developing brain tissue secretes an angiogenic factor that stimulates the proliferation of brain radial endothelial cells, suggesting that the proliferation and differentiation of neural precursors are involved in penetration of radial microvessels from leptomeningeal blood vessels into developing neural tissues (Risau, 1986). In this context, it should be pointed out that, in wild type tectum, radial glial cells in the neuroepithelial layer of the embryonic tectum express ADAM17 mRNA (Fig. 1). Their processes span the entire thickness of the tectum from the ventricular to the pial surface in a radial direction, which is the same direction as that of the penetrating microvessels. Provided that ADAM17 protein is present on the radial glial processes and ADAM17 may provide one of the molecular cues that promote radial sprouting of leptomeningeal blood vessels. The precise mechanism of ADAM17-induced proliferation of pericytes during brain microvessel development remains to be elucidated.

\section{Conclusions}

Our results demonstrated for the first time that the overexpression of ADAM17 in developing chicken brain promotes angiogenesis by increasing pericytes proliferation and capillary sprouting and results in morphological changes of the radial microvessels.

\section{Materials and Methods}

\section{Embryos and tissue preparation}

Fertilized eggs of White Leghorn chicken were obtained from a local farm and incubated in a forced-draft incubator (BSS160; Ehret, Emmendingen, Germany) at $37^{\circ} \mathrm{C}$ with $65 \%$ humidity. Chicken embryos were staged according to Hamburger and Hamilton (Hamburger and Hamilton, 1951). Embryos were studied at E1.5, E2, E2.5, E3, E4, E5, E6, E7, E8, E10, E12, E14, $\mathrm{E} 16, \mathrm{E} 18$, and E20 (at least 3 embryos at each stage). The older chicken embryos were killed by decapitation after deep anesthesia induced by cooling on ice. For RT-PCR analysis, the whole embryos, heads or brains were collected and placed immediately into liquid nitrogen and stored at $-80^{\circ} \mathrm{C}$. For in situ hybridization, brains were fixed in $4 \%$ formaldehyde solution on ice for 6 to $24 \mathrm{~h}$, depending on the size of the brains. Specimens were embedded in Tissue-Tec O.C.T. compound (Science Services, Munich, Germany), frozen in liquid nitrogen and stored at $-80^{\circ} \mathrm{C}$.

For transmission electron microscopy, the chicken brains were separated carefully after perfusion via the heart with $2.5 \%$ glutardialdehyde / $2 \%$ formaldehyde in $0.1 \mathrm{M}$ cacodylate buffer (Roth, Karlsruhe, Germany) for $5 \mathrm{~min}$. Subsequently, the tectum was isolated and fixed overnight with the perfusion solution at $4^{\circ} \mathrm{C}$, followed by fixation in $1 \%$ osmium tetroxide.

\section{Cloning of chicken ADAM17 cDNA}

Total RNA of chicken brain at E12 was extracted using TRIzol reagent according to the manufacturer's instruction (Invitrogen, Karlsruhe, Germany). The first strand of cDNA was synthesized in vitrousing the SuperScript FirstStrand Synthesis System (Invitrogen). PCR was performed to obtain the partial or full-length cDNA of chicken ADAM17 (GenBank:NM_001008682). The primers for the partial sequence including several exons of ADAM17 were 5'-tcccgcgtgctaaagttcctctgg-3' (upper) and 5'-ggcactcgcgctcctctcatcac-3' (lower), and for the full-length sequence were 5'-atgagactccggctgtggct-3' (upper) and 5'-tcagcactccgtctccttgc-3' (lower). After an initial denaturation step at $94^{\circ} \mathrm{C}$ for $2 \mathrm{~min}, \mathrm{PCR}$ was carried out with 30 cycles under the following conditions: denaturation at $94^{\circ} \mathrm{C}$ for $30 \mathrm{sec}$, annealing at $59^{\circ} \mathrm{C}$ for $30 \mathrm{sec}$ and extension at $68^{\circ} \mathrm{C}$ for $2.5 \mathrm{~min}$, followed by an extension at $68^{\circ} \mathrm{C}$ for $10 \mathrm{~min}$. The PCR products of partial sequence with a length of $1070 \mathrm{bp}$ and of full-length $2.5 \mathrm{~kb}$ sequence were cloned into pCRII-TOPO vector (Invitrogen) by the TA-cloning method. The positive clones containing the partial or full-length ADAM17 cDNAs were sequenced by a commercial service (MWG, Ebersberg, Germany). The full-length ADAM17 was further subcloned into a blunted EcoRI site in the eukaryotic expression vector pCAGGS. The pCAGGS-ADAM17 plasmid was used for overexpression of chicken ADAM17 protein by ex ovo electroporation. All plasmids were purified with the Maxi Plasmid Purification Kit (Qiagen, Hilden, Germany).

\section{Semi-quantitative RT-PCR}

Total RNA from whole E12 brain was prepared using the TRIzol reagent according to the manufacturer's instruction (Invitrogen). Semi-quantitative RT-PCR was performed using the One Step RT-PCR Kit (Qiagen). Glyceraldehyde-3-phosphate dehydrogenase (GAPDH) was used as an internal control to monitor the amount of RNA. The upper primer for GAPDH was 5'-gggctcatctgaagggtggtgcta-3' and the lower primer 5'-gtgggggagacagaagggaacaga-3' (expected product length of $810 \mathrm{bp}$ ). To obtain clear cDNA bands of both ADAM17 and GAPDH in one reaction, the ratio of the primer concentration between ADAM17 (primer for partial sequence) and GAPDH was adjusted to 5:1. The RT-PCR reaction was performed first at $50^{\circ} \mathrm{C}$ for $30 \mathrm{~min}$ for reverse transcription and at $95^{\circ} \mathrm{C}$ for $15 \mathrm{~min}$ for denaturation, followed by 30 cycles of amplification (denaturation at $94^{\circ} \mathrm{C}$ for $45 \mathrm{sec}$, annealing at $59^{\circ} \mathrm{C}$ for $45 \mathrm{sec}$ and extension at $72^{\circ} \mathrm{C}$ for $1.5 \mathrm{~min}$ ). The same amount of water instead of RNA was added in the reaction and served as a negative control. The PCR products were analyzed on a 1.5\% agarose gel, which was stained with ethidium bromide and photographed under UV light (BDA U-346; Biometra, Göttingen, Germany). The intensity of the individual bands was quantified with the software ImageQuant TL (Amersham Biosciences, Freiburg, Germany) and the relative amount was normalized to the corresponding GAPDH level.

\section{cRNA probe synthesis and in situ hybridization}

Digoxigenin-labeled sense and antisense cRNA probes were transcribed in vitro using the purified pCRII-TOPO plasmids containing the full-length or partial sequence of ADAM17 according to the manufacturer's instruction (Roche, Mannheim, Germany). Sense cRNA probe was used as negative controls for in situhybridization. Probes were purified by Quick Spin Column (Sephadex G50; Roche).

In situ hybridization on cryosection was performed according to a previously published protocol (Redies et al., 1993). Briefly, cryostat sections of $20 \mu \mathrm{m}$ thickness were fixed with $4 \%$ formaldehyde in phosphate-buffered saline and pretreated with proteinase $\mathrm{K}$ and acetic anhydride. Sections were hybridized overnight with cRNA probe at a concentration of about $3 \mathrm{ng} / \mathrm{ul}$ at $70^{\circ} \mathrm{C}$ in hybridization solution ( $50 \%$ formamide, $3^{\prime} \mathrm{SSC}, 10 \mathrm{mM}$ EDTA, $10 \%$ dextran sulfate, $1^{\prime}$ Denhardt's solution, $42 \mu \mathrm{g} / \mathrm{ml}$ yeast transfer RNA and $42 \mu \mathrm{g} / \mathrm{ml}$ salmon sperm DNA). After washing of the sections and removal of unbound CRNA by RNAse reaction, the sections were incubated with alkaline phosphatase-coupled anti-digoxigenin Fab fragments (Roche) overnight at $40 \mathrm{C}$. For visualization of the labeled mRNA, a substrate solution of nitroblue tetrazolium salt and 5-bromo-4-chloro-3-indoyl phosphate was added. The sections were viewed under a microscope (BX40; Olympus, Hamburg, Germany) and photographed with a digital camera (DP70; Olympus).

In order to identify anatomical structures, adjacent sections were stained with thionine according to a previously published protocol (Redies et al., 1993).

\section{Antibodies and immunohistochemistry}

The primary mouse monoclonal antibodies raised against alpha-smooth muscle actin (SMA; Sigma, Munich, Germany) (Skalli et al., 1986), green fluorescence protein (GFP; Sigma), proliferating cell nuclear antigen (PCNA; Abcam, Cambridge, UK), fibronectin (kind gift of R. Hynes, MIT, Cambridge) (Risau and Lemmon, 1988), laminin (31 and 3H11; DSHB, USA), and the rat polyclonal antibody against HT7 (kind gift of $\mathrm{H}$. Gerhardt, Institute of Pathology, University of Tübingen, Germany) (Bertossi et al., 2002) were used for immunostaining of sections in combination with appropriate Cy3or Alexa488-labeled secondary antibodies.

For immunofluorescent staining, the sections were first preincubated 
with skimmed milk solution ( $5 \%$ skimmed milk, $0.3 \%$ Triton $\mathrm{X}-100$, and $0.04 \%$ sodium azide in Tris-buffered saline, TBS) at room temperature for $60 \mathrm{~min}$ and then incubated with the primary antibody at $4^{\circ} \mathrm{C}$ overnight. In turn, an appropriate Cy3-labeled secondary antibody against species IgG was used at room temperature for 60 min. Finally, the dye Hoechst 33258 (Molecular Probes, Eugene, OR) was used for staining cell nuclei. Fluorescence was visualized and photographed under a fluorescence microscope (BX40, Olympus; or BZ-8000, Keyence Deutschland GmbH, Neu-Isenburg, Germany). For double immunostaining against PCNA and GFP, the sections were first heated in TBS by microwave for $10 \mathrm{~min}$ and cooled down at room temperature for $30 \mathrm{~min}$. Then immunostaining was performed according to a previously published protocol (Luo et al., 2006).

\section{Ex ovo electroporation}

Ex ovo electroporation was performed according to the protocol by Luo and Redies (2005). Briefly, fertilized eggs were cracked at about E2.5 and the embryos were transferred into a Petri dish system. Embryos incubated in shell-less culture until E6 were chosen for ex ovo electroporation. After the vitelline membrane was torn carefully with fine forceps over the tectum, a solution containing $2 \mu \mathrm{g} / \mu \mathrm{l}$ pCAGGS-ADAM17 plasmid and 0.25 $\mu \mathrm{g} / \mu \mathrm{l}$ pCAGGS-GFP plasmid (kind gift of Dr. H. Ogawa, National Institute of Basic Biology, Okazaki, Japan) mixed in Gey's buffered salt solution (GBSS; Invitrogen) was injected into the right tectal ventricle and electronic pulses ( $25 \mathrm{~V}, 60 \mathrm{~ms}$ pulse length, 6 pulses, $100 \mathrm{~ms}$ intervals) were applied immediately by an electroporator (CUY-21; Nepa Gene, Chiba, Japan). Transfected embryos were returned to the incubator until E12 and E14 for fixation and analysis.

\section{Cell apoptosis and proliferation analysis}

Cell apoptosis and proliferation were analyzed in the adjacent sections of the transfected and untransfected area of the electroporated embryos using the In situ Cell Death Detection Kit/TMR Red according to the manufacturer's instruction (Roche) and the antibody raised against the cell proliferation marker PCNA, respectively.

\section{Western blot analysis}

The expression of ADAM17 protein was estimated in lysates of human HEK cells by Werstern blot analysis after pCAGGS-GFP or pCAGGSADAM17 was transfected into HEK cells with LipofectamineTM 2000 kit (Invitrogen) according to the manufactory guidance. Protein concentrations were determined with a BCA protein assay (Pierce) with a photometer (Tecan, Crailsheim, Germany). Equal amounts of protein were loaded on 4-15\% SDS-polyacrylamide gels (BioRad, München, Germany) and electrophoresis was performed. The protein bands were then electrotransferred from gel onto nitrocellulose membrane (GE Healthcare, Freiburg, Germany). After the membrane is blocked with $3 \%$ milk in TBST Buffer for 1 hour, primary antibody against ADAM17 (Abcam ab57484, dilution 1:1000) and GAPDH (Abcam ab8245, dilution 1:10,000) is incubated with membrane at $4^{\circ} \mathrm{C}$ overnight. The following secondary antibodies were used: goat anti-rabbit Alexa Fluor 680, goat anti-mouse Alexa Fluor 680, and goat anti-mouse IRDye 800 (all from Invitrogen, 1:10,000). Detection was carried out using the Odyssey Infrared Imaging System (LI-COR Biosciences GmbH).

\section{Transmission electron microscopy}

Vibratome sections of chicken tectum were cut, dehydrated in ethanol and embedded in Durcupan ACM (Fluka, Buchs, Switzerland). After counterstaining with uranyl acetate and lead citrate according to the protocol previously described (Roberts, 2002), ultrathin sections were examined by an electron microscope (EM900; Zeiss, Jena, Germany) and photographed with a CCD digital camera (FastScan-F114; TVIPS, Gauting, Germany).

\section{Statistical analysis}

Aalytic data are presented as mean \pm SD or SEM at least three independent experiments. The ANOVA was used to estimate the significance of differences between cDNA bands measured by semi-quantitative RT-
PCR among distinct groups. Student's t-test was applied for evaluating significant differences between the numbers of the size and sprouts of the microvessels, of the pericytes, of the endothelial cells, and of the pixels of the vessel walls among different regions or groups. Differences were considered to be significant when the $P$-value was less than 0.05 .

\section{Acknowledgements}

We thank Dr. R. Hynes, Dr. H. Gerhardt, and Dr. H. Ogawa for kind gifts of reagents. This work was supported by a grant from the German Research Foundation (DFG, LU 1455/1-1).

\section{References}

BAUVOIS B. (2004). and Transmembrane proteases in cell growth and invasion: new contributors to angiogenesis? Oncogene 23: 317-329.

BERGERS G, andSONG S. (2005). The role of pericytes in blood-vessel formation and maintenance. Neuro Oncol 7: 452-464.

BERTOSSI M, GIROLAMO F, ERREDE M, BENAGIANO V, VIRGINTINO D and RONCALI L. (2002). Developmental changes of HT7 expression in the microvessels of the chick embryo brain. Anat Embryol (Berl) 205: 229-233.

BLOBEL CP. (2005). ADAMs: key components in EGFR signalling and development. Nat Rev Mol Cell Biol 6: 32-43.

CANAULT M, CERTEL K, SCHATZBERG D, WAGNER DD and HYNES RO. (2010) The lack of ADAM17 activity during embryonic development causes hemorrhage and impairs vessel formation. PLoS One 5: e13433.

CHOI YK and KIM KW. (2008). Blood-neural barrier: its diversity and coordinated cell-to-cell communication. BMB Rep 41: 345-352.

DOMESICK VB and MOREST DK. (1977). Migration and differentiation of ganglion cells in the optic tectum of the chick embryo. Neuroscience 2: 459-475.

DORE-DUFFY P. (2008). Pericytes: pluripotent cells of the blood brain barrier. Curr Pharm Des 14: 1581-1593.

EDWARDS DR, HANDSLEY MM and PENNINGTON CJ. (2009). The ADAM metalloproteinases. Mol Aspects Med 29: 258-289.

GERHARDTH, WOLBURG H and REDIES C. (2000). N-cadherin mediates pericyticendothelial interaction during brain angiogenesis in the chicken. Dev Dyn 218: $472-479$.

GODDARD DR, BUNNING RA and WOODROOFE MN. (2001). Astrocyte and endothelial cell expression of ADAM 17 (TACE) in adult human CNS. Glia 34: 267-271.

GÖOZ P, GÖOZ M, BALDYS A and HOFFMAN S. (2009). ADAM-17 regulates endothelial cell morphology, proliferation, and in vitro angiogenesis. Biochem Biophys Res Commun 380: 33-38.

HAMBURGER V and HAMILTON HL. (1951). A series of normal stages in the development of the chick embryo. Dev Dyn 195: 231-272.

KUKITA T, HATA K, KUKITA A and IIJIMA T. (1998) Laminin, a major basement membrane component of the blood vessel, as a negative regulator of osteoclastogenesis. Calcif Tissue Int 63: 140-142.

LAI CH and KUO KH. (2005). The critical component to establish in vitro BBB model: Pericyte. Brain Res Rev 50: 258-265.

LAVAIL JH and COWAN WM. (1971). The development of the chick optic tectum. I. Normal morphology and cytoarchitectonic development. Brain Res 28: 391-419.

Leibovich SJ, Polverini PJ, Shepard HM, Wiseman DM, Shively V, and Nuseir N Macrophage-induced angiogenesis is mediated by tumour necrosis factor-alpha. Nature 329: 630-632.

LUO J, JU MJ and REDIES C. (2006). Regionalized cadherin-7 expression by radia glia is regulated by Shh and Pax7 during chicken spinal cord development. Neuroscience 142: 1133-1143.

LUO $\mathrm{J}$ and REDIES C. (2005). Ex ovo electroporation for gene transfer into older chicken embryos. Dev Dyn 233: 1470-1477.

LUOJ, TREUBERT-ZIMMERMANNU and REDIESC. (2004). Cadherins guide migrating Purkinje cells to specific parasagittal domains during cerebellar development. Mol Cell Neurosci 25: 138-152.

NEHLS V, DENZERK and DRENCKHAHND. (1992). Pericyte involvement in capillary sprouting during angiogenesis in situ. Cell Tissue Res 270: 469-474.

OZERDEM U and STALLCUPWB. (2003). Early contribution of pericytes to angiogenic 


\section{J. Lin et al.}

sprouting and tube formation. Angiogenesis 6: 241-249.

Patan S. Vasculogenesis and angiogenesis as mechanisms of vascular network formation, growth and remodeling. $J$ Neurooncol 50: 1-15

REDIESC, ENGELHARTK Kand TAKEICHIM. (1993). Differential expression of N-and R-cadherin in functional neuronal systems and other structures of the developing chicken brain. J Comp Neurol 333: 398-416.

REINMUTH N, LIU W, JUNG YD, AHMAD SA, SHAHEEN RM, FAN F, BUCANA CD, MCMAHON G, GALLICK GE and ELLIS LM. (2001). Induction of VEGF in perivascular cells defines a potential paracrine mechanism for endothelial cell survival. FASEB J 15:1239-41.

RISAU W. (1986). Developing brain produces an angiogenesis factor. Proc Natl Acad Sci USA 83: 3855-3859.

RISAU W. (1997). Mechanisms of angiogenesis. Nature 386: 671-674.

RISAU W and LEMMON V. (1988). Changes in the vascular extracellular matrix during embryonic vasculogenesis and angiogenesis. Dev Biol 125: 441-450.

ROBERTS IM. (2002). Iso-butanol saturated water: a simple procedure for increasing staining intensity of resin sections for light and electron microscopy. $J$ Microsc 207: 97-107.
RONCALI L, NICO B, RIBATTI D, BERTOSSI M and MANCINI L. (1986). Effects of the chronic hypoxia on the developing blood-brain barrier in the chick embryo optic tectum. J Submicrosc Cytol 18: 537-541.

SAHIN U and BLOBEL CP. (2007). Ectodomain shedding of the EGF-receptor ligand epigen is mediated by ADAM17. FEBS Lett 581: 41-44.

SKALLIO, ROPRAZP, TRZECIAKA, BENZONANAG, GILLESSEND and GABBIANI G. (1986). A monoclonal antibody against alpha-smooth muscle actin: a new probe for smooth muscle differentiation. J Cell Biol 103: 2787-2796.

SWENDEMAN S, MENDELSON K, WESKAMP G, HORIUCHI K, DEUTSCH U, SCHERLE P, HOOPER A, RAFII S and BLOBEL CP. (2008). VEGF-A stimulates ADAM17-dependent shedding of VEGFR2 and crosstalk between VEGFR2 and ERK signaling. Circ Res 103: 916-918.

VAN HINSBERGH VW and KOOLWIJK P. (2008). Endothelial sprouting and angiogenesis: matrix metalloproteinases in the lead. Cardiovasc Res 78: 203-212.

WESKAMP G, MENDELSONK, SWENDEMANS, LE GALLS, MAY, LYMANS, HINOKI A, EGUCHI S, GUAIQUIL V, HORIUCHI K and BLOBEL CP. (2010) Pathological neovascularization is reduced by inactivation of ADAM17 in endothelial cells but not in pericytes. Circ Res 106: 932-940. 


\section{Further Related Reading, published previously in the Int. J. Dev. Biol.}

The seminal work of Werner Risau in the study of the development of the vascular system

Domenico Ribatti

Int. J. Dev. Biol. (2010) 54: 567-572

Notch in vertebrates - molecular aspects of the signal

Ken-Ichi Katsube and Kei Sakamoto

Int. J. Dev. Biol. (2005) 49: 369-374

Vasculogenesis and angiogenesis in the mouse embryo studied using quail/mouse chimeras

Michel Pudliszewski and Luc Pardanaud

Int. J. Dev. Biol. (2005) 49: 355-361

Vascular development: from precursor cells to branched arterial and venous networks

Anne Eichmann, Li Yuan, Delphine Moyon, Ferdinand leNoble, Luc Pardanaud and Christiane Bréant

Int. J. Dev. Biol. (2005) 49: 259-267

Astroglia-microvessel relationship in the developing human telencephalon

D Virgintino, E Maiorano, M Errede, A Vimercati, P Greco, L Selvaggi, L Roncali and M Bertossi

Int. J. Dev. Biol. (1998) 42: 1165-118

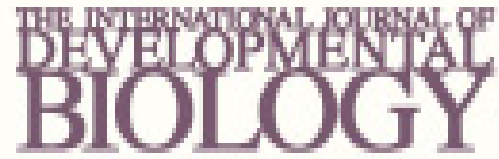

Whume st Fin ort
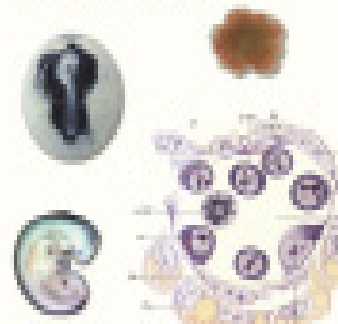

Apand thing
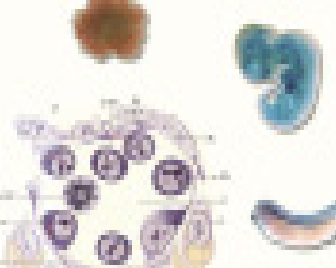

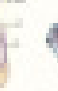
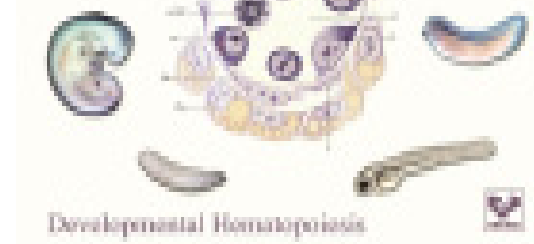

5 yr ISI Impact Factor $(2010)=2.961$
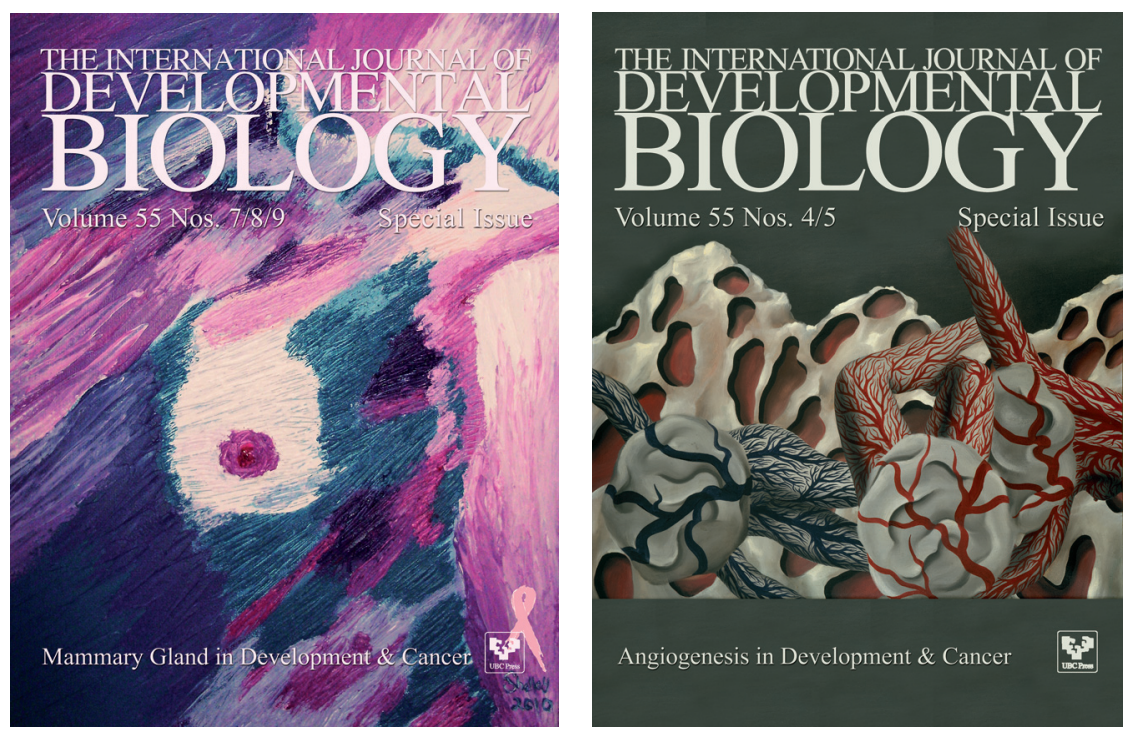